\title{
Health Care Economics
}

\section{Use of Midlevel Practitioners to Achieve Labor Cost Savings in the Primary Care Practice of an MCO}

\author{
Douglas W. Roblin, David H. Howard, Edmund R. Becker, \\ E. Kathleen Adams, and Melissa H. Roberts
}

Objective. To estimate the savings in labor costs per primary care visit that might be realized from increased use of physician assistants (PAs) and nurse practitioners (NPs) in the primary care practices of a managed care organization (MCO).

Study Setting/Data Sources. Twenty-six capitated primary care practices of a group model MCO. Data on approximately two million visits provided by 206 practitioners were extracted from computerized visit records for 1997-2000. Computerized payroll ledgers were the source of annual labor costs per practice from 1997-2000.

Study Design. Likelihood of a visit attended by a PA/NP versus MD was modeled using logistic regression, with practice fixed effects, by department (adult medicine, pediatrics) and year. Parameter estimates and practice fixed effects from these regressions were used to predict the proportion of $\mathrm{PA} / \mathrm{NP}$ visits per practice per year given a standard case mix. Least squares regressions, with practice fixed effects, were used to estimate the association of this standardized predicted proportion of PA/NP visits with average annual practitioner and total labor costs per visit, controlling for other practice characteristics.

Results. On average, PAs/NPs attended one in three adult medicine visits and one in five pediatric medicine visits. Likelihood of a PA/NP visit was significantly higher than average among patients presenting with minor acute illness (e.g., acute pharyngitis). In adult medicine, likelihood of a PA/NP visit was lower than average among older patients. Practitioner labor costs per visit and total labor costs per visit were lower $(p<.01$ and $p=.08$, respectively) among practices with greater use of PAs/NPs, standardized for case mix.

Conclusions. Primary care practices that used more PAs/NPs in care delivery realized lower practitioner labor costs per visit than practices that used less. Future research should investigate the cost savings and cost-effectiveness potential of delivery designs that change staffing mix and division of labor among clinical disciplines.

Key Words. Primary care, costs of care, midlevel practitioners, managed care

One strategy for managed care organizations (MCOs) to achieve cost savings is reconfiguration of division of labor so that care is aligned with a practitioner qualified to provide the care in a cost-effective manner. In primary care, MCOs can lower primary care delivery costs by making more effective use of 
midlevel practitioners (Flood et al. 1998; Scheffler, Waitzman, and Hillman 1996). Compensation of nurse practitioners (NPs) and physician assistants (PAs) is less than the compensation of physicians (MDs), and their training is appropriate for treatment of acute minor or stable chronic conditions, which are frequent in primary care, but are often attended by MDs.

Estimates of the proportion of primary care visits that might be attended by PAs or NPs range between 50 percent and 75 percent (Crandall et al. 1984; Frampton and Wall 1994; Hooker and Freeborn 1991; Spitzer et al. 1974). One survey of expert panels concluded that midlevel practitioners were capable of attending up to 90 percent of primary care visits (Rabin and Spector 1980). The percent of patients in national surveys who receive at least some primary care from midlevel practitioners is lower than these estimates, although the percent has been increasing in recent years as representation of PAs/NPs in the practitioner workforce expands (Hooker and McCaig 2001; Druss et al. 2003).

Numerous studies concerning the outcomes of primary care visits have been rather consistent in finding comparable patient satisfaction or quality of care on visits attended by either PAs/NPs or MDs (reviews by Brown and Grimes 1995; Horrocks, Anderson, and Salisbury 2002; Sox 1979). In contrast, there are few published estimates of the cost savings to be expected from integration of PAs/NPs into a primary care practice.

Most studies have reported that use of midlevel practitioners in primary care can lower service delivery costs (Greenfield et al. 1978; Grzybicki et al. 2002; Hershey and Kropp 1979; Holmes, Livingston, and Mills 1976; Hooker 2002; LeRoy 1981; Venning et al. 2000). At least one study, however, has concluded that use of specially trained nurses was not cost saving (Spector et al. 1975). Some of these estimates of cost-saving potential are dated; others are based on either a single practice or single midlevel practitioner. Reporting of costs (per visit, per episode, per patient) differs from study to study. Little attention has been given to the impact on cost-savings estimates of the casemix differences of patients attended by PAs/NPs compared with those

The Garfield Memorial Fund of the Kaiser Permanente Medical Care Program has provided funds for this project.

Address correspondence to Douglas Roblin, Ph.D., Research Department, Kaiser Permanente, 3495 Piedmont Rd. NE, Bldg. 9, Atlanta, GA 30305. Melissa H. Roberts, M.S., is also with the Research Department at Kaiser Permanente. David H. Howard, Ph.D., Edmund R. Becker, Ph.D., and E. Kathleen Adams, Ph.D., are with the Department of Health Policy and Management, Rollins School of Public Health, Emory University, Atlanta, GA. 
attended by MDs. Although the argument for integrating PAs/NPs into primary care is essentially predicated on their labor cost savings, few studies (Venning et al. 2000; Hooker 2002) separate cost savings specifically due to labor cost differentials from other costs (principally costs of ancillary services due to practice variation).

Valid, generalizable evidence on the economic impact of PA/NP integration is essential in order to evaluate the impact of alternative primary care delivery system designs on service costs. We evaluated the use of midlevel practitioners in 26 capitated primary care practices of a mid-sized MCO from 1997 to 2000 . Our study had three objectives: (1) characterize the case mix of visits attended by PAs/NPs compared with those attended by MDs, (2) compute a measure of predicted use of PAs/NPs for a standard case mix, and (3) estimate the association between this predicted PA/NP use and average labor cost savings per visit across the practices.

\section{METHODS}

\section{Study Setting}

Kaiser Permanente Georgia (KPG) is a group and network model MCO providing medical services to approximately 275,000 members throughout the metropolitan Atlanta area. In 1997, this MCO reorganized its group model primary care delivery system (88 percent of the membership) into semiautonomous, self-directed primary care practices (10 in the pediatric medicine department, 16 in the adult medicine department). The prototype practice consists of a practitioner group (two to three MDs, one to two PAs/ $\mathrm{NPs}$ ) and a support staff. Adult medicine practices provide care to approximately 10,000-12,000 empanelled members, and pediatric medicine practices to approximately $5,000-8,000$ members.

Each practice has a management team (lead MD and lead RN) and has latitude in how it adapts MCO resources (e.g., scheduling systems, disease registries) to the needs of its empanelled members. A practice receives a budget for staffing that is determined by a capitation payment and the number of its empanelled members. A practice has flexibility in staffing, and autonomy in its division of labor, as long as it remains within this budget and MCO personnel guidelines.

Redesign of the primary care delivery system into self-directed, semiautonomous practices was intended to increase the number of visits that could be provided at a relatively constant budget, thereby lowering the cost 
per primary care visit. Increased use of PAs/NPs was integral to this objective. Implementation of this strategy involved: reconfiguration of division of labor between PAs/NPs (with emphasis on acute minor or stable chronic illness) and MDs (with emphasis on complex acute or unstable chronic illness), appropriate triage of visits between $\mathrm{PAs} / \mathrm{NPs}$ and $\mathrm{MDs}$, and increased representation of PAs/NPs in the practitioner workforce. The ultimate goal was a primary care practitioner workforce consisting of 50 percent PAs/NPs and 50 percent MDs.

Each KPG member selects or, in absence of a selection, is assigned to a primary care practice and primary care physician (PCP). A member may change practice or PCP at any time. During regular office hours (8 A.M.-6 P.M. weekdays), a member requests an appointment by directly calling the practice to which he or she is empanelled. The practice advice nurse assesses the patient need for care, any request for a specific practitioner, and the availability of both the requested and other practitioners. Appointments are typically made on the day of the call or the following day. About 50 percent of patients request a specific practitioner and 75 percent of those visits are booked with the requested practitioner.

\section{Study Population and Period}

The study population consists of the 26 primary care practices comprising the group model delivery system from 1997 through 2000. During this time, physicians or midlevel practitioners in the adult medicine department attended 1,445,420 visits, and physicians or midlevel practitioners in the pediatric medicine department attended 694,571 visits.

\section{DATA AND MEASURES}

\section{Primary Care Visits}

Primary care visits are defined as visits occurring in either the adult medicine or pediatric medicine departments during regular office hours. Visits included those attended by practice physicians or midlevel practitioners as well as pool practitioners or locum tenens (combined approximately 6-7 percent of visits) temporarily employed by a practice. Study visits occurred between January 1997 and December 2000.

A computerized record is completed at the time of a visit. This record identifies the patient, practice, attending practitioner, and role (MD, PA, NP), and precoded ICD-9-CM codes, which allow the practitioner to identify 
medical conditions characterizing the visit. Each record allows for up to 3 ICD-9-CM diagnosis codes (with 75 percent of records having only one code).

Eleven classes of presenting conditions were defined from the first three digits of the ICD-9-CM codes: hypertension (401), diabetes (250), asthma (493), otitis media $(380,381,382)$, rhinitis $(472,477)$, sinusitis $(461,473)$, pharyngitis (462), acute URI (465), bronchitis (466, 490, 491), neck or back pain $(723,724)$, and joint pain $(719,729)$. These specific groups of codes represented the most frequent acute minor illness or chronic conditions attended in primary care. Visits not meeting these criteria were defined as "Other." Because hypertension and diabetes are infrequent presenting conditions in pediatric medicine, these two classes were recoded to "Other." Classes of presenting conditions were coded as binary variables for each visit.

\section{Patient Demographics}

For each patient with a primary care visit, patient date of birth and gender were obtained from computerized enrollment records and linked with the visit records by unique patient identifiers. Age of the patient was computed as of December 31 of the year of the visit and classified into age groups. For adult medicine, the age groups were: $3-17,18-29,30-39,40-54,55-64,65-74$, 75 years and older. For pediatric medicine, the age groups were: $0-2,3-12$, $13-17$ years.

\section{Practitioner and Staff Characteristics}

Computerized timesheets were obtained for 1997-2000. Each timesheet identifies the employee and hours worked at a practice. Number of full-time equivalents (FTEs) by role (MD, PA/NP) per practice year were estimated by summing hours worked with the practice and dividing by a standard number of hours worked in a year by a full-time employee $(2,080)$. Hours for leave (e.g., sick, vacation) were enumerated on timesheets and counted toward definition of FTEs.

Initial date of employment of practitioners with KPG was obtained from staffing databases. Mean practitioner tenure for a practice during a year was computed as the average of tenure of all staff practitioners with the practice as of December 31 of that year.

\section{Labor Costs}

Computerized payroll ledgers were obtained for 1997-2000. The ledgers itemized annual compensation by role (MD, PA/NP, RN, LPN/LVN, medical 
assistant, and receptionist) for each practice during each of the four study years. Compensation included wages and benefits of permanent employees as well as costs of temporary internal (pool) or contract employees (e.g., locum tenens physicians). Compensation is reconciled with hours worked per practice, ensuring that, in the aggregate, labor costs per practice year represent hours worked with a practice during the year.

Average annual practitioner labor cost per visit was defined as the total practitioner (MD, PA/NP) labor cost for a practice in a year divided by the total MD and PA/NP visits in the practice during the year. Average annual total labor cost per visit was defined as the total practitioner and support staff (RN, LPN/LVN, medical assistant, and receptionist) labor cost for a practice in a year divided by the total MD and $\mathrm{PA} / \mathrm{NP}$ visits in the practice in the year.

\section{ANALYSIS}

For each of the two departments (adult medicine, pediatrics), we described the association of practitioner type attending the visit with patient age, gender, presenting condition, and visit year. Practitioner attending the visit was defined from the visit record. Association was assessed with a $\chi^{2}$ test of independence.

Our overall study objective was to analyze the association between extent of use of PAs/NPs in primary care delivery and visit labor costs, adjusted for practice case mix. To accomplish this objective, we used a threestep modeling strategy.

First, as a function of case mix, we estimated the likelihood that a PA/NP would attend a visit. One logistic regression, with fixed effects for practices, was estimated for each of the four years within the two departments (total of eight regressions). The dependent variable was whether a PA/NP (versus $\mathrm{MD}$ ) attended the visit. Independent variables were the classes of presenting conditions and patient age group and gender. Practice fixed effects in the models account for the unobserved characteristics of practices that do not vary over time and affect PA/NP use through factors other than patient case mix.

We estimated models separately by year within department for several reasons. One, a practice fixed effect averaged over four years would fail to represent the changes in $\mathrm{PA} / \mathrm{NP}$ use within a practice over time. Two, our principal outcome-labor costs per visit-was measured at the level of practice year. This aligned the results obtained from the first stage (propensity of a practice to use $\mathrm{PAs} / \mathrm{NPs}$ ) and our principal outcome of interest (labor costs per visit) at the same level of observation-the practice year. 
Second, we used the results of this first step to compute the proportion of visits predicted to be attended by PAs/NPs in a practice in a year for a standard population. The standard population was based on all primary care visits in the department for a year. We computed the probability that each of these visits would be attended by a PA/NP for a practice in the year using the standard population (i.e., presenting condition, patient age, and gender), the parameter estimates from the logistic regression for the department for the year, and the practice fixed effects for the year. The proportion of visits predicted to be attended by PAs/NPs in a practice in a year was the average of the individual visit probabilities. This proportion measured variation in propensities of practices in a department in a year to use PAs/NPs holding case mix constant. This step produced a dataset with 104 observations: 26 practices, four years for each practice.

Finally, we estimated the association between average annual labor costs per visit per practice and this predicted proportion, adjusted for other practice characteristics. One least squares regression, with fixed effects for practices, was estimated for average annual practitioner labor costs per visit and another for average annual total labor costs per visit. Independent variables in the model included the proportion of visits predicted to be attended by PAs/NPs in the practice for the year, number of visits provided, practitioner average tenure, department (pediatrics or adult medicine), and year.

Our principal hypothesis was that average annual labor costs per visit would significantly $(\alpha=.05)$ vary with the propensity of a practice to use PAs/ NPs and would be negative in direction. Total number of visits was included to adjust for economies of scale. Tenure was included to account for the incremental increases in wages and benefits that accrue from continued service, on average, with KPG. Year was included to capture annual inflationary trends and other unmeasured changes in cost structure over the four-year study period.

$S A S$ software (version 8.02; 2001) was used for general data management and descriptive statistics. MLWin (version 1.1) (Rasbash et al. 2000) was used to estimate random effects regression models.

\section{RESULTS}

Overall, PAs/NPs attended 32.4 percent of adult medicine primary care visits (Table 1). Adult medicine patients presenting with acute pharyngitis or acute URI were most frequently attended by PAs/NPs (55.1 percent and 45.0 
Table 1: Distribution of Primary Care Visits* and Percent of Visits Attended by PAs/NPs

\begin{tabular}{|c|c|c|c|c|}
\hline & \multicolumn{2}{|c|}{ Adult Medicine } & \multicolumn{2}{|c|}{ Pediatric Medicine } \\
\hline & $\begin{array}{l}\text { Percent of } \\
\text { Visits }\end{array}$ & $\begin{array}{l}\text { Percent Attended } \\
\text { by PAs/NPs }\end{array}$ & $\begin{array}{l}\text { Percent of } \\
\text { Visits }\end{array}$ & $\begin{array}{l}\text { Percent Attended } \\
\text { by PAs/NPs }\end{array}$ \\
\hline $\begin{array}{l}\text { Overall Percent of Visits } \\
\text { Attended by PAs/NPs }\end{array}$ & - & 32.4 & - & 18.5 \\
\hline \multicolumn{5}{|l|}{ Presenting Condition $^{\dagger}$} \\
\hline Hypertension & 8.0 & 15.4 & - & - \\
\hline Asthma & 1.2 & 28.6 & 3.7 & 16.1 \\
\hline Diabetes & 3.8 & 29.0 & - & - \\
\hline Otitis media/Otitis externa & 2.4 & 41.4 & 12.5 & 19.4 \\
\hline Rhinitis (allergic and chronic) & 2.9 & 34.5 & 2.3 & 18.2 \\
\hline Sinusitis (acute and chronic) & 6.3 & 43.1 & 3.9 & 22.4 \\
\hline Acute pharyngitis & 2.3 & 55.1 & 5.0 & 23.8 \\
\hline Acute URI & 5.0 & 45.0 & 9.5 & 18.8 \\
\hline Bronchitis (acute and chronic) & 2.8 & 37.9 & 1.8 & 17.6 \\
\hline Neck or back pain & 4.7 & 29.2 & 0.4 & 14.2 \\
\hline Joint or limb pain & 4.8 & 30.7 & 0.7 & 16.1 \\
\hline Other & 55.9 & 31.5 & 60.1 & 17.8 \\
\hline \multicolumn{5}{|l|}{ Patient Age $\left(\right.$ years) ${ }^{\dagger}$} \\
\hline 0-2 (3-17 for adult medicine) & 0.4 & 32.1 & 34.7 & 18.3 \\
\hline $3-12$ & & & 46.3 & 18.5 \\
\hline $13-17$ & & & 19.0 & 19.0 \\
\hline $18-29$ & 16.1 & 44.3 & & \\
\hline 30-39 & 25.3 & 38.9 & & \\
\hline $40-54$ & 37.3 & 30.5 & & \\
\hline $55-64$ & 11.8 & 22.3 & & \\
\hline $65-74$ & 6.1 & 15.1 & & \\
\hline 75 and older & 3.0 & 11.8 & & \\
\hline \multicolumn{5}{|l|}{ Patient Gender $^{\dagger}$} \\
\hline Female & 60.4 & 33.6 & 48.7 & 19.9 \\
\hline Male & 39.6 & 30.4 & 51.3 & 17.2 \\
\hline \multicolumn{5}{|l|}{ Visit Year $^{\dagger}$} \\
\hline 1997 & 24.2 & 30.4 & 27.0 & 18.5 \\
\hline 1998 & 25.3 & 33.1 & 25.8 & 16.0 \\
\hline 1999 & 25.4 & 33.0 & 24.4 & 16.7 \\
\hline 2000 & 25.2 & 33.0 & 22.8 & 23.3 \\
\hline
\end{tabular}

*1,445,420 adult medicine visits, 694,571 pediatric medicine visits.

${ }^{\dagger}$ Indicates that percent of visits attended by PAs/NPs versus MDs varies significantly with patientvisit characteristics (presenting condition, patient age, patient gender, visit year) for $p<.01$ among both adult medicine and pediatric medicine practices.

percent of visits for those conditions, respectively), and those presenting with hypertension (15.4 percent) least frequently. Younger patients were much more likely than older patients to have their visit attended by a PA/NP. For 
example, among patients 18 to 29 years of age, 44.3 percent of visits were attended by PAs/NPs; however, among patients 75 and older, PAs/NPs attended only 11.8 percent of visits. The percent of primary care visits attended by PAs/NPs increased from 1997 to 1998 but remained relatively constant from 1998 through 2000.

On average, PAs/NPs attended 18.5 percent of pediatric medicine visits from 1997 to 2000 (Table 1). Pediatric medicine patients presenting with acute pharyngitis or sinusitis were most frequently attended by PAs/NPs (23.8 percent and 22.4 percent of visits for these conditions, respectively), asthma (16.1 percent) least frequently. The percent of primary care visits attended by PAs/ NPs decreased from 1997 to 1998 and then increased from 1998 through 2000.

Results of the first-stage logistic regressions for likelihood of the primary care visit being attended by a PA/NP compared with an MD for 1997 and 2000 in the two departments are displayed in Table 2 (1998 and 1999 models available upon request). ${ }^{1}$ Adjusted for case mix, practice fixed effects were relatively normally distributed about the median intercept estimate for each of the eight models (graphs available on request). Given the large numbers of visits, intraclass coefficients (ICCs) were moderate (.058 to .140), indicating clustering of propensity to use PAs/NPs among practices. ${ }^{2}$

Overall, the adjusted odds ratios (OR) for the patient-level covariates were consistent with the variation in percents of visits attended by PAs/NPs by patient age, gender, and presenting condition (Table 1). Among adult medicine practices, visits for acute minor illness were significantly more likely, and chronic conditions less likely, to be attended by PAs/NPs than MDs. For example, PAs/NPs were most likely to attend visits in 2000 for pharyngitis (adjusted OR of 2.141) or URI (adjusted OR of 1.866), and least likely to attend visits for hypertension (adjusted OR of 0.625). In pediatric medicine, PAs/NPs were most likely to attend visits in 2000 for pharyngitis $(\mathrm{OR}=1.251)$, and least likely to attend visits for neck or back pain ( $\mathrm{OR}=0.732$ ).

Among visits to adult medicine practices, the likelihood that a visit was attended by a PA/NP declined significantly with patient age, controlling for presenting condition. For example, the adjusted ORs in 2000 for 55-64-yearold patients, 65-74-year-old patients, and 75 and older patients were 0.392, 0.237 , and 0.177 , respectively, relative to $18-29$-year-old patients.

From 1997 through 2000, the likelihood that a PA/NP or MD attended a visit changed for specific visit types, notably in adult medicine (Table 2). For example, adjusted ORs for the likelihood that a PA/NP attended a visit for acute pharyngitis increased from 1.702 to $2.141(p<.05)$, while the adjusted ORs for diabetes visits attended by PAs/NPs decreased from 2.112 to 0.932 
Table 2: Adjusted Odds Ratios for Visit Attended by PA/NP versus MD Estimated from Random Intercept Logistic Regression of Visit Nested within Practice

\begin{tabular}{|c|c|c|c|c|}
\hline & \multicolumn{4}{|c|}{ Adjusted Odds Ratios } \\
\hline & \multicolumn{2}{|c|}{ Adult Medicine } & \multicolumn{2}{|c|}{ Pediatric Medicine } \\
\hline & 1997 & 2000 & 1997 & 2000 \\
\hline \multicolumn{5}{|l|}{ Presenting Condition } \\
\hline Hypertension & $0.565^{*}$ & $0.625^{*}$ & - & - \\
\hline Asthma & $0.725^{*}$ & 0.996 & 0.961 & 0.910 \\
\hline Diabetes & $2.112^{*}$ & $0.932^{*}$ & - & - \\
\hline Otitis (media, externa) & $1.303^{*}$ & $1.560^{*}$ & $1.154^{*}$ & $1.195^{*}$ \\
\hline Rhinitis (allergic, acute) & $1.171^{*}$ & $1.279^{*}$ & $0.970 *$ & $1.085^{*}$ \\
\hline Sinusitis (acute, chronic) & $1.368^{*}$ & $1.589^{*}$ & $1.117^{*}$ & $1.244^{*}$ \\
\hline Pharyngitis & $1.702^{*}$ & $2.141^{*}$ & $1.502^{*}$ & $1.251^{*}$ \\
\hline URI & $1.399^{*}$ & $1.866^{*}$ & $0.892^{*}$ & $1.157^{*}$ \\
\hline Bronchitis (acute, chronic) & $1.110^{*}$ & $1.465^{*}$ & $0.807^{*}$ & 1.018 \\
\hline Neck or back pain & $0.750 *$ & $1.078^{*}$ & $0.748^{*}$ & $0.732 *$ \\
\hline Joint or limb pain & $0.836^{*}$ & $1.114^{*}$ & $0.839^{*}$ & $0.834^{*}$ \\
\hline \multicolumn{5}{|l|}{ Patient Age (years) } \\
\hline $0-2$ (3-17 for adult medicine) & $0.693^{*}$ & $0.535^{*}$ & $0.936^{*}$ & $0.915^{*}$ \\
\hline $3-12$ & & & 0.974 & $0.937 *$ \\
\hline $30-39$ & $0.826^{*}$ & $0.826^{*}$ & & \\
\hline $40-54$ & $0.631^{*}$ & $0.599 *$ & & \\
\hline $55-64$ & $0.458^{*}$ & $0.392^{*}$ & & \\
\hline $65-74$ & $0.334 *$ & $0.237 *$ & & \\
\hline 75 and older & $0.248^{*}$ & $0.177 *$ & & \\
\hline \multicolumn{5}{|l|}{ Gender } \\
\hline Female & $1.124^{*}$ & $1.145^{*}$ & $1.148^{*}$ & $1.194^{*}$ \\
\hline \multicolumn{5}{|l|}{ Model Statistics } \\
\hline ICC & 0.076 & 0.058 & 0.140 & 0.101 \\
\hline Hosmer-Lemeshow & $139.08^{\ddagger}$ & $67.69^{\ddagger}$ & $81.97^{\ddagger}$ & $130.01^{\ddagger}$ \\
\hline Model chi-square & $13941.3^{\ddagger \ddagger}$ & $24237.5^{\ddagger \ddagger}$ & $998.4^{\ddagger \ddagger}$ & $516.1^{\ddagger \ddagger}$ \\
\hline Number of visits & 349,138 & 363,882 & 187,826 & 157,995 \\
\hline
\end{tabular}

*Indicates adjusted odds ratio is significantly different from 1.000 for $p<.05$.

${ }^{\ddagger}$ Significant for $p<.01, d f=8$.

${ }^{\ddagger}$ Significant for $p<.01, d f=18$ for the adult medicine models, and 12 for the pediatric medicine models.

'Reference is: Other condition, 18-29 years for adult medicine and 13-17 years for pediatric medicine, and male.

$(p<.05) .^{3}$ Results from pediatrics medicine were mixed. Adjusted ORs for the likelihood that a PA/NP attended a visit for URI increased from 0.892 to 1.157 $(p<0.05)$; however, adjusted ORs for acute pharyngitis visits attended by PAs/NPs decreased from 1.502 to $1.251(p<.05)$. 
The proportion of $\mathrm{PA} / \mathrm{NP}$ visits predicted per practice year, given a standard case mix, varied widely across the practices (Table 3, expressed as a percent). Among adult medicine practices, the interquartile range of the predicted percent of $\mathrm{PA} / \mathrm{NP}$ visits was 24.9 percent to 39.0 percent per practice year. Similarly, among pediatric medicine practices, the interquartile range was 12.8 percent to 24.1 percent.

Variation in use of PAs/NPs in care delivery was principally a consequence of the level of their employment in the practices. PAs/NPs as a percent of practitioner FTEs were positively correlated with both observed PA/NP visits as a percent of practice visits $(\rho=.937$ in adult medicine, $\rho=.924$ in pediatric medicine, both $p<.01)$ and predicted PA/NP visits given

Table 3: PA/NP Use, Practice Staff Mix, and Primary Care Labor Costs by Department, 1997-2000

\begin{tabular}{|c|c|c|c|c|}
\hline & \multirow[b]{2}{*}{$\begin{array}{l}\text { Mean of Practice- } \\
\text { Year Values }\end{array}$} & \multicolumn{3}{|c|}{ Distribution of Practice-Year Values } \\
\hline & & $\begin{array}{l}\text { Seventy-fifth } \\
\text { Percentile }\end{array}$ & Median & $\begin{array}{c}\text { Twenty-fifth } \\
\text { Percentile }\end{array}$ \\
\hline \multicolumn{5}{|l|}{ Adult Medicine (64 Practice Years) } \\
\hline Predicted percent of PA/NP visits & $32.5 \%$ & $39.0 \%$ & $31.3 \%$ & $24.9 \%$ \\
\hline Observed percent of $\mathrm{PA} / \mathrm{NP}$ visits & $31.9 \%$ & $40.1 \%$ & $30.9 \%$ & $23.5 \%$ \\
\hline \multicolumn{5}{|l|}{ Average practitioner FTEs } \\
\hline MD & 2.85 & 3.25 & 2.73 & 2.36 \\
\hline $\mathrm{PA} / \mathrm{NP}$ & 1.33 & 1.82 & 1.23 & 0.92 \\
\hline $\begin{array}{l}\text { PAs/NPs as percent of } \\
\text { practitioner FTEs }\end{array}$ & $31.5 \%$ & $39.5 \%$ & $30.9 \%$ & $23.5 \%$ \\
\hline \multicolumn{5}{|l|}{ Average labor costs per visit } \\
\hline Practitioner & $\$ 23.74$ & $\$ 26.49$ & $\$ 23.93$ & $\$ 21.53$ \\
\hline Total & $\$ 36.02$ & $\$ 39.35$ & $\$ 36.66$ & $\$ 33.34$ \\
\hline Visits $(1,000 \mathrm{~s})$ & 24.584 & 22.850 & 21.471 & 20.322 \\
\hline Average practitioner tenure (years) & 4.3 & 5.4 & 4.1 & 3.2 \\
\hline \multicolumn{5}{|l|}{ Pediatric Medicine (40 Practice Years) } \\
\hline Predicted percent of $\mathrm{PA} / \mathrm{NP}$ visits & $19.5 \%$ & $24.1 \%$ & $21.2 \%$ & $12.8 \%$ \\
\hline Observed percent of $\mathrm{PA} / \mathrm{NP}$ visits & $17.5 \%$ & $23.6 \%$ & $20.4 \%$ & $12.1 \%$ \\
\hline \multicolumn{5}{|l|}{ Average practitioner FTEs } \\
\hline MD & 2.78 & 3.39 & 2.76 & 2.25 \\
\hline $\mathrm{PA} / \mathrm{NP}$ & 0.73 & 0.99 & 0.83 & 0.04 \\
\hline $\begin{array}{l}\mathrm{PAs} / \mathrm{NPs} \text { as percent of } \\
\text { practitioner FTEs }\end{array}$ & $19.1 \%$ & $26.5 \%$ & $23.5 \%$ & $1.6 \%$ \\
\hline \multicolumn{5}{|l|}{ Average labor costs per visit } \\
\hline Practitioner & $\$ 28.04$ & $\$ 29.49$ & $\$ 27.45$ & $\$ 25.82$ \\
\hline Total & $\$ 42.25$ & $\$ 45.57$ & $\$ 40.60$ & $\$ 37.54$ \\
\hline Visits $(1,000 s)$ & 17.365 & 20.517 & 16.978 & 12.744 \\
\hline Average practitioner tenure (years) & 6.7 & 7.8 & 6.9 & 5.7 \\
\hline
\end{tabular}


a standard case mix $(\rho=.912$ in adult medicine and $\rho=.905$ in pediatrics medicine, both $p<.01)$.

Labor costs per primary care visit averaged about $\$ 24$ for practitioner labor and $\$ 36$ for total labor per practice year in adult medicine (Table 3). Labor costs per visit on pediatric medicine practices were higher than those on adult medicine practices. There was substantial clustering of average annual labor costs per visit among the practices (ICCs of .524 and .489; Table 4). ${ }^{4}$

Adjusted for patient case mix, practices that more extensively used PAs/ NPs in care delivery had lower average practitioner labor costs $(p<.01)$ and total labor costs per visit $(p=.08$; Table 4$)$. For example, an adult medicine practice at or above the seventy-fifth percentile of observed to expected ratio of visits attended by PAs/NPs realized practitioner labor cost savings of at least $\$ 1.44$ per visit (6.1 percent of the adult medicine average) and total labor cost savings of at least $\$ 1.11$ per visit (3.1 percent of the adult medicine average) compared with an adult medicine practice at or below the twenty-fifth

Table 4: Parameter Estimates for Average Labor Costs per Visit per Practice Year Estimated from Random Intercept Linear Regression of Year Nested within Practice

\begin{tabular}{|c|c|c|c|c|}
\hline & \multicolumn{4}{|c|}{ Per Visit Costs } \\
\hline & \multicolumn{2}{|c|}{ Practitioner Costs } & \multicolumn{2}{|c|}{ Total Costs } \\
\hline & $\beta$ & $P$ & $\beta$ & $P$ \\
\hline Intercept & $\$ 26.18$ & $<.01$ & $\$ 38.57$ & $<.01$ \\
\hline $\begin{array}{l}\text { Predicted percent of PA/NP visits } \\
\text { (per } 10 \% \text { increments)** }\end{array}$ & -1.02 & $<.01$ & -0.79 & .08 \\
\hline $\begin{array}{l}\text { Visits per practice year (per } 1,000 \\
\text { visit increments) }\end{array}$ & -0.11 & .10 & -0.26 & .01 \\
\hline Adult medicine & -3.19 & $<.01$ & -4.89 & $<.01$ \\
\hline Mean practitioner tenure (per year) ${ }^{\dagger}$ & 0.45 & .06 & 0.54 & .08 \\
\hline 1998 & 0.84 & .18 & 1.85 & .04 \\
\hline 1999 & 0.91 & .17 & 3.43 & $<.01$ \\
\hline 2000 & 2.97 & $<.01$ & 6.13 & $<.01$ \\
\hline ICC & \multicolumn{2}{|c|}{0.524} & \multicolumn{2}{|c|}{0.489} \\
\hline Model chi-square & \multicolumn{2}{|c|}{$63.3^{\ddagger}$} & \multicolumn{2}{|c|}{$94.1^{\ddagger}$} \\
\hline Number of practice years & \multicolumn{2}{|c|}{104} & \multicolumn{2}{|c|}{104} \\
\hline
\end{tabular}

*Expected percent per practice year computed using the logistic regression parameter estimates by year, the population case mix for the year, and the practice-specific propensity to use PAs/NPs in primary care delivery.

'Variables are centered at the practice means (Table 3).

${ }^{\ddagger}$ Significant for $p<.01, d f=7$. 
percentile. Because pediatrics medicine visits were, on average, more costly, and the range of predicted $\mathrm{PA} / \mathrm{NP}$ use was smaller, the labor cost savings on those visits was lower $(\$ 1.15$, or 4.1 percent for practitioner labor costs per visit, $\$ 0.89$ or 2.1 percent for total labor costs per visit). Additional labor cost savings per visit accrued to practices with higher visit volumes $(p=.01$ for total labor costs). Although the significance of the effects was marginal, labor cost savings were attenuated among practices with greater years of practitioner tenure ( $p=.06$ for practitioner labor costs, $p=.08$ for total labor costs).

\section{DISCUSSION}

In this $\mathrm{MCO}$, primary care visits were actively triaged between PAs/NPs and MDs according to patients' presenting conditions. Patients presenting with acute minor illnesses (e.g., pharyngitis, URI, sinusitis) were typically more likely to have their care provided by PAs/NPs than MDs. Conversely, patients presenting with chronic conditions were typically more likely to have their care provided by MDs than PAs/NPs.

The PAs/NPs attended primary care visits generally in proportion to their FTE representation in the practitioner workforce. On average, about one in three adult medicine visits and about one in five pediatrics medicine visits was attended by a PA/NP. Similarly, about one in three adult medicine practitioner FTEs was a $\mathrm{PA} / \mathrm{NP}$ and about one in five pediatrics medicine practitioner FTEs was a PA/NP.

Average labor costs per visit provided by the practices varied with the extent to which PAs/NPs were used in care delivery. Practices that more extensively used PAs/NPs in providing care realized lower labor costs per visit than practices that made less extensive use of PAs/NPs. Average annual practitioner labor costs per visit for an adult medicine practice at the seventyfifth percentile of $\mathrm{PA} / \mathrm{NP}$ use were 6.1 percent lower, and average annual total labor costs per visit were 3.1 percent lower than an adult medicine practice at the twenty-fifth percentile. For pediatric medicine practices, the savings were 4.1 percent and 2.1 percent, respectively.

Estimates of labor cost savings per primary care visit in this $\mathrm{MCO}$ are similar to estimates that can be computed from the several studies that differentiate labor costs from total costs. Labor cost savings per visit in a primary care practice computed from data reported by Venning et al. (2000) are approximately 8-9 percent ${ }^{5}$; those computed from data reported by Hooker (2002) range from 5 percent to 9 percent. $^{6}$ 
Although such estimated labor cost savings per visit may appear to be inconsequential (several dollars per visit), the net savings to an MCO are substantial when accumulated across many thousands of visits per year. Several hundreds of thousands of dollars per year, for example, might be used productively by expanding patient education or chronic disease management programs (to promote healthy lifestyles or avert future morbidity).

Given the potential cost savings that obtain from PA/NP use in primary care delivery in this $\mathrm{MCO}$, why do the practices vary so widely in $\mathrm{PA} / \mathrm{NP}$ employment? When the practices were constituted in 1997, PA/NP representation in each practice was essentially determined by PA/NP representation at the MCO's nine primary care clinics. If a clinic had few or no PAs/NPs practicing primary care, the newly constituted practices had few or none. Subsequent circumstances have affected the ability of most practices to transform their practitioner mix. One, expansion of practitioner staff, specifically hiring of PAs/NPs, was constrained by slow membership growth from 1997 to 2000. Two, opportunities to convert MD FTEs to PA/NP FTEs in a practice were limited by relatively low practitioner turnover during 19972000. When a practice added PA/NP FTEs, they were deployed similarly (e.g., attending visits for acute pharyngitis) to practices in which PAs/NPs represented a large proportion of practitioner FTEs.

The experience of this $\mathrm{MCO}$, and this analysis of the potential for labor cost savings to be achieved from PAs/NPs into primary care delivery, has implications for other MCOs.

First, the potential practitioner labor cost savings per visit depends on the compensation differential between MDs and PAs/NPs. In this MCO in 2000, a PA/NP was compensated at approximately 50 percent of an internal medicine MD. In other MCOs, the compensation differential may be less, thereby decreasing the potential savings in practitioner labor costs from what we estimated in this study. Also, as the responsibilities and experience of PAs/ NPs increase over time, the compensation differential between PAs/NPs and MDs may narrow and further diminish practitioner labor cost saving potential. In fact, in this $\mathrm{MCO}$, the $\mathrm{PA} / \mathrm{NP}$ compensation per FTE increased from approximately 38 percent of MD compensation per FTE (1997) to 50 percent.

Second, in primary care practices where PAs/NPs and MDs work in similar circumstances and have similar visit rates, total practice labor costs will be increased in proportion to the number of practitioner FTEs supported. Total labor cost savings per visit were less, on average, than practitioner cost savings per visit. Hiring of midlevel practitioners allows a capitated primary care practice to increase visit capacity, however, staff must be hired to support 
the additional administrative and clinical activities surrounding the visit. For example, if three staff FTEs support one practitioner FTE, then a practice with two MD FTEs will require six support staff FTEs whereas a practice with one MD FTE and two PA/NP FTEs will require nine support staff-even though the practitioner labor costs are equivalent (assuming a PA/NP FTE is compensated at 50 percent of an MD FTE).

Third, use of midlevel practitioners into primary care delivery will depend on the practice environment and the extent to which that use is accepted and actively supported. In this MCO, medical group leadership fully supports the multidisciplinary practice model. Recent debates about the role of midlevel practitioners in primary care and whether the role is "good" for physicians suggests that support of a multidisciplinary practice model is generally less accepted elsewhere (Kassirer 1994; Mundinger 1994). Underuse of midlevel practitioners has been attributed to physician resistance (Fottler 1979; Freeborn and Hooker 1995). A key challenge to effective role integration in multidisciplinary teams is creating a practice environment in which physicians and midlevel practitioners perceive each as complements to one another, rather than as substitutes for one another (Grumbach and Coffman 1998). While the argument for integrating PAs/NPs into primary care is essentially predicated on their labor cost savings, practice variation and its impact on variable costs (principally costs of ancillary services due to practice variation) need to be considered in assessing MCO-level cost implications. Labor cost savings might be attenuated in the aggregate if PAs/ NPs order other services at higher rates than MDs. Evidence as to this point is mixed. Some studies have found higher rates of use or costs of ancillary services associated with visits attended by PAs/NPs than visits attended by MDs (Hemani et al. 1999; Hooker 2002; Venning et al. 2000). Other studies have found no significant difference in ancillary services use or return visits (Shum et al. 2000; Kinnersley et al. 2000). Several observational studies of prescribing of antibiotics on visits for treatment of infectious disease found no differences in rates of antibiotic prescribing between nurse practitioners and those attended by physicians (Butler et al. 2001; Cox and Jones 2000). In this MCO, evidence as to whether PAs/NPs used ancillary services at higher rates than MDs was similarly equivocal (data available upon request).

Our study has various strengths. The scope of practice was representative of most primary care practices, including a broad range of minor acute illnesses and chronic conditions. The large number of visits and the four-year study period allowed for relatively stable estimates of visit triage. Availability 
of detailed visit data permitted adjustment for case mix on labor costs. The study covered 26 capitated practices and large numbers of practitioners were represented: $68 \mathrm{PAs} / \mathrm{NPs}$ and $140 \mathrm{MDs}$. The PAs/NPs practice in similar circumstances and have similar daily appointment rates. The primary care practices were characterized by wide variation in integration of PAs/NPs: from less than 10 percent to nearly 50 percent of practitioner FTEs. Both the patient population and practitioners include large proportions of women and minorities (principally African American-45 percent of patients and 40 percent of practitioners).

Generalizability of our study's results may be limited. This was an observational study. Overall, our analyses describe outcomes, on average, from $\mathrm{PA} / \mathrm{NP}$ use in one $\mathrm{MCO}$-not what could or should occur ideally or in other MCOs. Those MCOs with less compensation differential between PAs/ NPs and MDs or less use of PAs/NPs in care delivery would be expected to realize less cost savings per visit than we estimated for this MCO. Although conclusions were based on large numbers of visits and practitioners and casemix differences between PAs/NPs and MDs were taken into account, other unmeasured variables might account for some of the cost savings potential of $\mathrm{PA} / \mathrm{NP}$ use. Our estimates of the cost saving potential of PA/NP use are based on a linear model, which may not be a realistic assumption.

In conclusion, MCOs throughout the United States are under pressure to manage cost inflation while improving patient outcomes. The scope of primary care physician practice has expanded in recent years, as specialty care has been shifted into primary care (St. Peter et al. 1999). The number of recommended preventive services and their frequency is increasing (Yarnall et al. 2003). Concerns have been raised about physician workload and stress and their effects on patient care.

A primary care delivery model that integrates midlevel practitioners into the practice presents one strategy to respond to the competing demands of controlling costs, maintaining or improving access, and expanding scope of care. The PAs/NPs can attend to many of the acute minor illnesses that patients present in primary care and can provide many preventive services, allowing physicians to concentrate their attention on complex patients. Further cost saving changes in care delivery could involve nurses within the practice, many of whom are competent to provide time-intensive patient instruction, such as in chronic disease self-care techniques or telephone followup. While physicians are needed to develop, review, and modify treatment plans, other clinical staff can frequently and appropriately provide specific services and procedures in a potentially cost-beneficial manner. 


\section{NOTES}

1. While the Hosmer-Lemeshow statistic (Hosmer and Lemeshow 1989) suggests a suboptimal fit of the model's predicted probabilities to observed probabilities by decile, the magnitude of these statistics is quite low given the large number of observations on which these $\chi^{2}$ statistics are based. Inspection of the observed-toexpected ratio by decile suggested no bias in over- or underprediction at either end of the decile range.

2. The ICCs were estimated from a random intercepts-only model, with practice fixed effects.

3. Differences in parameter estimates between 1997 and 2000 tested for significance using the formula: $\left.z=\left(\beta_{2000}-\beta_{1997}\right) /\left(\operatorname{var}\left(\beta_{2000}\right)+\operatorname{var}\left(\beta_{1997}\right)\right) / 2\right)^{1 / 2}$.

4. The ICCs were estimated from a random intercepts-only model, with practice fixed effects.

5. Venning et al. (2000; Table 6) report average labor costs per NP visit of $£ 11.71$ and per MD visit of $£ 14.14$. We assume a $50 / 50$ percent visit distribution between NPs and MDs (insofar as the study was conducted as a randomized trial). Savings in labor costs per primary care visit for this visit distribution, compared with labor costs for a practice in which only MDs attended, are computed as: $(1-((£ 11.71 \times 0.50)+$ $(£ 14.14 \times 0.50)) / £ 14.14) \times 100 \%=8.6 \%$

6. Hooker (2002) reports average labor costs per episode attended by PA or MD (Figure 2) and the visit distribution by episode (Figure 1). We assume the visit distribution between PAs and MDs is representative of the practice studied. Per episode savings in labor costs per primary care visit for this visit distribution, compared with labor costs for a practice in which only MDs attended, are computed as:

For bronchitis: $(1-((\$ 92.23 \times 0.17)+(\$ 133.63 \times 0.83)) / \$ 133.63) \times 100 \%=5.3 \%$

For otitis media: $(1-((\$ 83.29 \times 0.22)+(\$ 140.07 \times 0.78)) / \$ 140.07) \times 100 \%=8.9 \%$

\section{REFERENCES}

Brown, S. A., and D. E. Grimes. 1995. "A Meta-analysis of Nurse Practitioners and Nurse Midwives in Primary Care." Nursing Research 44 (6): 332-9.

Butler, C. C., M. Rees, P. Kinnersley, S. Rollnick, and K. Hood. 2001. "A Case Study of Nurse Management of Upper Respiratory Tract Infections in General Practice." Journal of Advanced Nursing 33 (3): 328-33.

Cox, C., and M. Jones. 2000. "An Evaluation of the Management of Patients with Sore Throats by Practice Nurses and GPs." British Journal of General Practice 50 (460): 872-6.

Crandall, L. A., W. P. Santulli, M. L. Radelet, K. E. Kilpatrick, and D. E. Lewis. 1984. "Physician Assistants in Primary Care: Patient Assignment and Task Delegation." Medical Care 22 (3): 268-82.

Druss, B. G., S. C. Marcus, M. Olfson, T. Tanielian, and H. A. Pincus. 2003. "Trends in Care by Nonphysician Clinicians in the United States." Journal of the American Medical Association 348 (2): 130-7. 
Flood, A. B., A. M. Fremont, K. Jin, D. M. Bott, J. Ding, and R. C. Parker Jr. 1998. "How Do HMOs Achieve Savings? The Effectiveness of One Organization's Strategies." Health Services Research 33 (1): 79-99.

Fottler, M. D. 1979. "Physician Attitudes toward Physician Extenders: A Comparison of Nurse Practitioners and Physician Assistants." Medical Care 17 (5): 536-49.

Frampton, J., and J. S. Wall. 1994. "Exploring the Use of NPs and PAs in Primary Care." HMO Practice 8 (4): 165-70.

Freeborn, D. K., and R. S. Hooker. 1995. "Satisfaction of Physician Assistants and Other Nonphysician Providers in a Managed Care Setting." Public Health Reports 110 (6): 714-9.

Greenfield, S., A. L. Komaroff, T. M. Pass, H. Anderson, and S. Nessim. 1978. "Efficiency and Cost of Primary Care by Nurses and Physician Assistants." New England Journal of Medicine 298 (6): 305-9.

Grumbach, K., and J. Coffman. 1998. "Physicians and Nonphysician Clinicians: Complements or Competitors?" Editorial. Journal of the American Medical Association 280 (9): 825-6.

Grzybicki, D. M., P. J. Sullivan, J. M. Oppy, A.-M. Bethke, and S. Raab. 2002. "The Economic Benefit for Family/General Practices Employing Physician Assistants." American Journal of Managed Care 8 (7): 613-20.

Hemani, A., D. A. Rastegar, C. Hill, and M. S. al-Ibrahim. 1999. "A Comparison of Resource Utilization in Nurse Practitioners and Physicians." Effective Clinical Practice 2 (6): 258-65.

Hershey, J. C., and D. H. Kropp. 1979. “A Re-appraisal of the Productivity Potential and Economic Benefits of Physician's Assistants." Medical Care 17 (6): 592-606.

Holmes, G., G. Livingston, and E. Mills. 1976. "Contribution of a Nurse Clinician to Office Practice Productivity: Comparison of Two Solo Primary Care Practices.” Health Services Research 11 (1): 21-33.

Hooker, R. S. 2002. “A Cost Analysis of Physician Assistants in Primary Care.” Journal of the American Academy of Physician Assistants 15 (11): 39-42, 45, 48 passim.

Hooker, R. S., and D. K. Freeborn. 1991. "Use of Physician Assistants in a Managed Health Care System.” Public Health Reports 106 (1): 90-4.

Hooker, R. S., and L. F. McCaig. 2001. "Use of Physician Assistants and Nurse Practitioners in Primary Care." Health Affairs 20 (4): 231-8.

Horrocks, S., E. Anderson, and C. Salisbury. 2002. "Systematic Review of Whether Nurse Practitioners Working in Primary Care Can Provide Equivalent Care to Doctors.” British Medical Journal 324 (7341): 819-23.

Hosmer, D. W., and S. Lemeshow. 1989. Applied Logistic Regression. New York: Wiley. Kassirer, J. P. 1994. "What Role for Nurse Practitioners In Primary Care?" Editorial. New England Journal of Medicine 330 (3): 204-5.

Kinnersley, P., E. Anderson, K. Parry, J. Clement, L. Archard, P. Turton, A. Stainthorpe, A. Fraser, C. C. Butler, and C. Rogers. 2000. "Randomised Controlled Trial of Nurse Practitioner versus General Practitioner Care for Patients Requesting 'Same Day' Consultations in Primary Care.” British Medical Journal 320 (7241): 1043-8.

LeRoy, L. 1981. The Costs and Effectiveness of Nurse Practitioners: OTA Case Studies of Medical Technologies, no. 16. Washington, DC: Office of Technology Assessment. 
Mundinger, M. O. 1994. "Advanced-Practice Nursing: Good Medicine for Physicians? [Sounding Board]." New England Journal of Medicine 330 (3): 211-4.

Rabin, D. L., and K. K. Spector. 1980. "Delegation Potential of Primary Care Visits by Physician Assistants, Medex and Primex." Medical Care 18 (11): 1114-25.

Rasbash, J., W. Browne, H. Goldstein, M. Yang, J. Plewis, M. Healy, G. Woodhouse, D. Draper, I. Langford, and T. Lewis. 2000. MLWin (version 1.1). London: Institute of Education, University of London.

SAS Institute. 2001. SAS/STAT statistical software (version 8.2). Cary, NC: SAS Institute.

Scheffler, R. M., N. J. Waitzman, and J. M. Hillman. 1996. "The Productivity of Physician Assistants and Nurse Practitioners and Health Work Force Policy in the Era or Managed Health Care." Journal of Allied Health 25 (3): 207-17.

Shum, C., A. Humphreys, D. Wheeler, M. A. Cochrane, S. Skoda, and S. Clement. 2000. "Nurse Management of Patients with Minor Illnesses in General Practice: Multicentre, Randomized Controlled Trial.” British Medical Journal 320 (7241): $1038-43$.

Sox, H. C. Jr. 1979. "Quality of Patient Care by Nurse Practitioners and Physician's Assistants: A Ten-Year Perspective.” Annals of Internal Medicine 91 (3): 459-68.

Spector, R., P. McGrath, J. Alpert, P. Cohen, and H. Aikins. 1975. "Medical Care by Nurses in an Internal Medicine Clinic: Analysis of Quality and Its Cost.” Journal of the American Medical Association 232 (12): 1234-7.

Spitzer, W. O., D. L. Sackett, J. C. Sibley, R. S. Roberts, M. Gent, D. J. Kergin, B. C. Hackett, and A. Olynich. 1974. "The Burlington Randomized Trial of the Nurse Practitioner." New England Journal of Medicine 290 (5): 251-6.

St. Peter, R.F, M. C. Reed, P. Kemper, and D. Blumenthal. 1999. "Changes in the Scope of Care Provided by Primary Care Physicians." New England Journal of Medicine 341 (26): 1980-5.

Venning, P., A. Durie, M. Roland, C. Roberts, and B. Leese. 2000. "Randomized Controlled Trial Comparing Cost Effectiveness of General Practitioners and Nurse Practitioners in Primary Care.” British Medical Journal 320 (7241): 104853.

Yarnall, K. S., K. I. Pollak, T. Østbye, K. M. Krause, and J. L. Michener. 2003. "Primary Care: Is There Enough Time for Prevention?” American Journal of Public Health 93 (4): 635-41. 
626 